\title{
The Association of Aging with White Matter Integrity and Functional Connectivity Hubs
}

\author{
Albert C. Yang 1,2,3,4*, Shih-Jen Tsai ${ }^{2,3}$, Mu-En Liu ${ }^{2,3}$, Chu-Chung Huang ${ }^{5}$ and \\ Ching-Po Lin ${ }^{5 *}$ \\ ${ }^{1}$ Division of Interdisciplinary Medicine and Biotechnology, Beth Israel Deaconess Medical Center/Harvard Medical School, \\ Boston, MA, USA, ${ }^{2}$ Department of Psychiatry, Taipei Veterans General Hospital, Taipei, Taiwan, ${ }^{3}$ Division of Psychiatry, \\ School of Medicine, National Yang-Ming University, Taipei, Taiwan, ${ }^{4}$ Center for Dynamical Biomarkers and Translational \\ Medicine, National Central University, Chungli, Taiwan, ${ }^{5}$ Institute of Brain Science, National Yang-Ming University, Taipei, \\ Taiwan
}

OPEN ACCESS

Edited by:

Rodrigo Orlando Kuljiš, University of Miami, USA

Reviewed by:

J. Arturo García-Horsman, University of Helsinki, Finland Franziska Matthäus, University of Heidelberg, Germany

*Correspondence: Albert C. Yang cyang1@bidmc.harvard.edu Ching-Po Lin chingpolin@gmail.com

Received: 28 January 2016 Accepted: 30 May 2016 Published: 15 June 2016

Citation:

Yang AC, Tsai S-J, Liu M-E, Huang C-C and Lin C-P (2016) The Association of Aging with White Matter Integrity and Functional Connectivity Hubs. Front. Aging Neurosci. 8:143. doi: 10.3389/fnagi.2016.00143
Normal aging is associated with reduced cerebral structural integrity and altered functional brain activity, yet the association of aging with the relationship between structural and functional brain changes remains unclear. Using combined diffusion tensor imaging (DTI) and functional magnetic resonance imaging (fMRI) modalities, we hypothesized that aging-related changes in white matter integrity (i.e., fractional anisotropy) was associated with the short- or long-range functional connectivity density (FCD) in hub regions. We tested this hypothesis by using a healthy aging cohort comprised of 140 younger adults aged 20-39 years and 109 older adults aged 60-79 years. Compared with the younger group, older adults exhibited widespread reductions in white matter integrity with selective preservation in brain stem tracts and the cingulum connected to the hippocampus and cingulate cortex, whereas FCD mapping in older adults showed a reduced FCD in the visual, somatosensory, and motor functional networks and an increased FCD in the default mode network. The older adults exhibited significantly increased short- or long-range FCD in functional hubs of the precuneus, posterior, and middle cingulate, and thalamus, hippocampus, fusiform, and inferior temporal cortex. Furthermore, DTI-fMRI relationship were predominantly identified in older adults in whom short- and long-range FCD in the left precuneus was negatively correlated to structural integrity of adjacent and nonadjacent white matter tracts, respectively. We also found that long-range FCD in the left precuneus was positively correlated to cognitive function. These results support the compensatory hypothesis of neurocognitive aging theory and reveal the DTI-fMRI relationship associated with normal aging.

Keywords: aging, diffusion tensor imaging, functional connectivity density mapping, compensatory hypothesis

\section{INTRODUCTION}

Aging is associated with remarkable changes in brain structure and function. Studies have revealed that, compared with younger adults, older people exhibit reduced brain size, and increased ventricular volume (Scahill et al., 2003), loss of neuronal body or synaptic density (Morrison and Hof, 1997), loss of gray matter intensity (Good et al., 2001), or reduced white matter integrity (Pagani et al., 2008). However, research with blood oxygen level dependent (BOLD) 
signals from functional magnetic resonance imaging (fMRI; Fox and Raichle, 2007) has identified a disturbed functional connectivity associated with aging (see Ferreira and Busatto, 2013 for reviews). Nevertheless, the relationship between structural and functional brain changes associated with aging remains under explored (see Minati et al., 2007; Bennett and Rypma, 2013 for reviews).

Because transmission of neuronal signals relies on physical connections between a myriad of neurons via axons and synapses, neural activity is thought to be modulated through structural connections (Koch et al., 2002; Boorman et al., 2007; Greicius et al., 2009). Furthermore, the quality of structural connections, or white matter integrity, has been suggested to influence functional connectivity of gray matter brain regions (Bennett and Rypma, 2013). In this context, combining structural and fMRI techniques is appropriate for examining the relationship between brain structure and functional changes associated with aging. Whereas, diffusion tensor imaging (DTI) can be used to quantify white matter structural integrity, such as fractional anisotropy (FA) value, resting-state BOLD fMRI can reveal functional dependency among different brain regions.

Previous studies examining the effect of aging on the relationship between structural integrity and functional connectivity have suggested that patterns vary between younger and older adults (Bennett and Rypma, 2013). White matter integrity has been positively correlated to functional activity in younger adults (Forstmann et al., 2008; Kim and Whalen, 2009; Van Eimeren et al., 2010), but negatively correlated to functional activity in older adults (Persson et al., 2006; Madden et al., 2007). However, other studies have shown both patterns (Baird et al., 2005; Koch et al., 2010) or opposite directions (Putnam et al., 2008; De Chastelaine et al., 2011) of DTI-fMRI correlation, relative to the findings of previously mentioned studies. Studies on aging regarding the DTI-fMRI relationship have predominantly been based on various cognitive tasks (Bennett and Rypma, 2013), and the effect of aging on the relationship between structural integrity and resting-state functional connectivity is unclear. Such an investigation could facilitate clarifying the neurocognitive mechanism in aging, particularly in the default mode network (DMN) brain regions.

Therefore, this study investigated the association of aging with structural integrity and resting-state functional connectivity in a healthy aging cohort. We focused on highly connected hub regions of functional networks based on functional connectivity density (FCD) mapping (Tomasi and Volkow, 2010, 2012a). This study tested the hypotheses that aging-related changes in structural integrity was associated with alterations in shortor long-range functional connectivity in hub regions, and that this association predominantly occurs in older adults to compensate for the structural decline associated with normal aging.

\section{MATERIALS AND METHODS}

\section{Participants}

The study cohort comprised 249 Han Chinese participants recruited from communities in Northern Taiwan. Among them,
140 participants aged 20-39 years comprised the younger group (mean: $28.5 \pm 4.9$ years; male/female: 67/73), and 109 participants aged 60-79 years comprised the older group (mean: $67.4 \pm 6.2$ years; male/female: 52/57). The study constituted a continuing effort of the Healthy Aging Project (Yang et al., 2013, 2014), and received approval from the Institutional Review Board of Taipei Veterans General Hospital. Each participant gave written informed consent and was evaluated by a trained research assistant using the Mini-International Neuropsychiatric Interview to exclude the presence of Axis I psychiatric disorders (Sheehan et al., 1998). All participants were assessed for cognitive function, using the Mini-Mental State Examination (MMSE; Folstein et al., 1975) and the Wechsler Digit Span Task (Wechsler, 1997). Older participants were further assessed using the Clinical Dementia Rating Scale (CDR) (Hughes et al., 1982) to exclude dementia (CDR $>0$ ). Overall exclusion criteria for all participants were as follows: (a) presence of dementia; (b) presence of Axis I psychiatric disorders, such as schizophrenia, bipolar disorders, or unipolar depression; and (c) a history of neurological conditions, such as head injury, stroke, or Parkinson's disease. Demographic characteristics of age groups are shown in Table 1.

\section{Image Acquisition}

We performed fMRI scanning at National Yang-Ming University, using a 3.0T Siemens MRI scanner (Siemens Magnetom Tim Trio, Erlangen, Germany) equipped with a 12-channel head coil. The scanning protocol was consistent with our prior reports (Yang et al., 2013, 2014). For resting-state image scanning, T2*weighted images with BOLD contrast were measured using a gradient echo-planar imaging (EPI) sequence (repetition time TR $=2500 \mathrm{~ms}$, echo time $\mathrm{TE}=27 \mathrm{~ms}$, FoV $=200 \mathrm{~mm}$, flip angle $=$ $77^{\circ}$, matrix size $=64 \times 64$, voxel size $=3.44 \times 3.44 \times 3.40 \mathrm{~mm}$ ). For each run, 200 EPI volume images were acquired along the AC-PC plane. For structural image scanning, whole-brain DTI images were acquired using a single-shot spin-echo EPI sequence in the axial-plane with the following parameters: repetition time/echo time $=11,000 / 104 \mathrm{~ms}$; number of excitations $=3$; matrix size $=$ $128 \times 128$; field of view $=26 \mathrm{~cm}$; slice thickness $=2.0 \mathrm{~mm} ; 70$ slices; $b$-value $=1000 \mathrm{~s} / \mathrm{mm}^{2} ; 30$ isotropic diffusion directions and three non-diffusion weighted T2 images.

TABLE 1 | Demographic characteristics in different age groups.

\begin{tabular}{lcccc}
\hline Variables & $\begin{array}{c}\text { Younger } \\
(\mathbf{N}=\mathbf{1 4 0})\end{array}$ & $\begin{array}{c}\text { Older } \\
(\mathbf{N}=\mathbf{1 0 9})\end{array}$ & $\boldsymbol{t}$ or $\boldsymbol{x}$ & $\boldsymbol{P}$ \\
\hline Age, year & $28.5 \pm 4.9$ & $67.5 \pm 6.2$ & -54.9 & $<0.001$ \\
Sex, male & $67(47.9)$ & $52(47.7)$ & 0.01 & n.s. \\
Handedness, right & $133(95.0)$ & $105(96.3)$ & 0.04 & n.s. \\
Mini mental state & $29.2 \pm 1.1$ & $27.6 \pm 2.5$ & 6.8 & $<0.001$ \\
examination & & & & \\
Digit forward task & $15.3 \pm 1.4$ & $13.2 \pm 2.2$ & 8.7 & $<0.001$ \\
$\begin{array}{l}\text { Digit backward } \\
\text { task }\end{array}$ & $10.8 \pm 2.9$ & $6.9 \pm 3.3$ & 9.8 & $<0.001$ \\
& & & &
\end{tabular}

Categorical data are given as number (\%). n.s., non-significant. 


\section{Structural and Resting Imaging Processing}

Resting BOLD image data were preprocessed and analyzed using SPM8 (Wellcome Department of Imaging Neuroscience, London, UK) implemented in MATLAB (Mathworks Inc., Sherborn, MA, USA). BOLD data were slice-timing corrected, realigned, and normalized into the standard stereotaxic space of the Montreal Neurological Institute (MNI) EPI template, and resampled to a 3-mm cubic voxel. Covariates of BOLD time series were regressed out before FCD analysis was performed, including the time courses of six head motions, white matter, and cerebrospinal fluid. No global signal regression was performed to avoid introducing distortions in the time series data (Murphy et al., 2009; Anderson et al., 2011). All participants included in this study exhibited a maximal displacement of $<1.5 \mathrm{~mm}$ at each axis and an angular motion of $<1.5^{\circ}$ for each axis. The first five data points $(12.5 \mathrm{~s})$ in any BOLD time series were discarded because of the instability of initial MRI scanning, leaving 195 data points in the final data. Temporal low-pass filtering $(0.01-0.08 \mathrm{~Hz})$ was performed to reduce the influence of high-frequency noise from physiologic confounders.

Raw DTI images were preprocessed using the Functional Magnetic Resonance Imaging of the Brain (FMRIB) diffusion toolbox (Raz et al., 2007), part of the Functional Magnetic Resonance Imaging of the Brain software library (FSL; Morrison and Hof, 1997). The raw DTI image was corrected for the effects of head movement and eddy currents. A brain extraction tool was used to remove all non-brain parts of the image (Good et al., 2001). From these images, fractional anisotropy (FA) values were calculated by fitting a tensor model to the data at each voxel. The Tract-Based Spatial Statistics (TBSS, part of FSL) pipeline (Scahill et al., 2003) was then used to register and normalize FA images to the MNI standard space used to target the FMRIB58 FA standard-space image. A mean FA image was created and thinned to create a mean FA skeleton that represented the centers of all tracts common to the group. An FA threshold of 0.2 was used to exclude non-skeletal voxels (Scahill et al., 2003). The aligned FA data of each subject was then projected onto this skeleton, and the resulting data were subjected to subsequent statistical analyses.

\section{Functional Connectivity Density Mapping}

FCD mapping (Tomasi and Volkow, 2010, 2012a) was developed based on resting-state BOLD signals for mapping whole-brain short-range and long-range functional connectivity with high spatial resolution (3-mm isotropic), which allows identifying functional hubs (Tomasi and Volkow, 2010, 2011). Tomasi et al. defined three types of FCD measure: global FCD, short-range FCD, and long-range FCD.

For each gray matter voxel, global FCD was defined as the number of global functional connections, $\mathrm{k}\left(\mathrm{x}_{i}\right)$, determined through Pearson correlations between BOLD signals at voxel $\mathrm{x}_{i}$ and those in the remaining gray matter voxels using an arbitrary threshold $r>0.6$ (Tomasi and Volkow, 2010). Short-range FCD was defined as local neighbors of voxel $\mathrm{x}_{i}$, which was computed using a growth algorithm to identify voxels that were adjacent to a voxel linked to $\mathrm{x}_{i}$ on a continuous path, and had connectivity strength $r>0.6$ with voxel $\mathrm{x}_{i}$. The growth algorithm was repeated in an iterative manner until no new neighbors could be found.

In the final step, the long-range FCD of voxel $x_{i}$ was computed by subtracting global FCD and short-range FCD. The calculation of FCD was computed for all gray matter voxels. The whole-brain short- and long-range FCD maps were further normalized by the whole-brain mean FCD, and were spatially smoothed with an 8-mm Gaussian kernel in SPM8 to minimize differences in the functional anatomy of the brain across subjects (Tomasi and Volkow, 2012a).

\section{Statistical Analysis}

Figure 1 illustrates the analysis flow of image analyses. Statistical analyses of parametric imaging data were conducted using MATLAB. First, regional differences in whole-brain parametric mapping (i.e., short-range and long-range FCD and DTI-FA) between age groups were examined using the general linear model. Second, we determined the anatomical regions of interest (ROIs) based on 48 white matter tract labels defined in the ICBMDTI-81 atlas developed at Johns Hopkins University (Minati et al., 2007; Bennett and Rypma, 2013). The averaged FA of

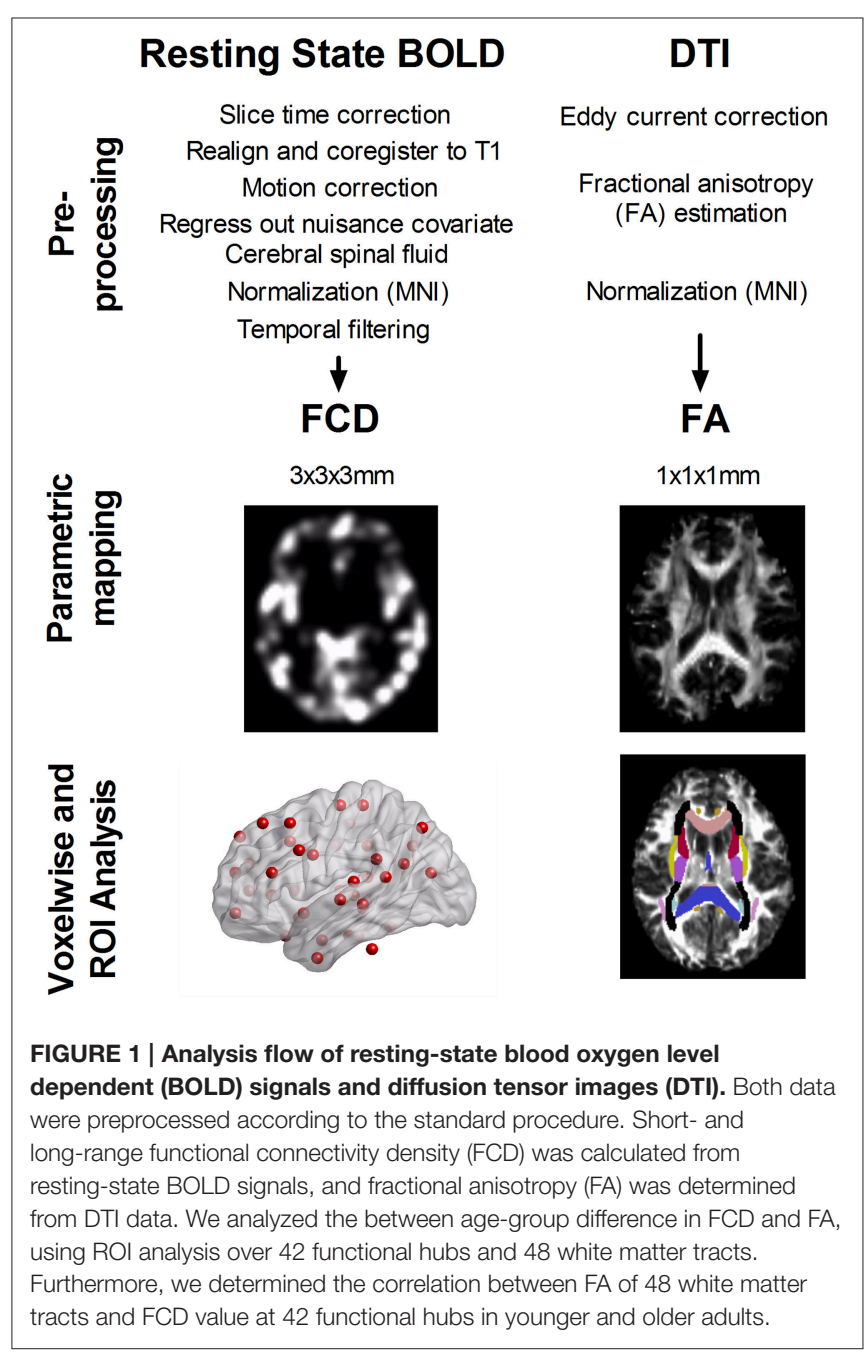


each white matter tract was calculated by overlaying the groupspecific white matter skeleton and the ICBM-DTI-81 atlas with a specific white matter labeling (Van Eimeren et al., 2010). The strength of the short- and long-range FCD were similarly calculated from 42 functional hubs identified previously (Tomasi and Volkow, 2012a). We used a cubic mask containing 27 voxels centered on hub coordinates to extract the average FCD values. A Student's $t$-test was used to assess between-group difference in ROI measures. These FCD and FA measures were additionally used to assess the correlation between FA and FCD values derived from gray and white matter ROIs at the group level, respectively. This approach determined the extent to which the FCD of the gray matter hub can be explained in a group of subjects by using the mean FA value of a given white matter tract, controlling for the effect of age and sex. Hence, we performed a partial correlation to control for age and sex and conducted the analyses separately in the younger and older group. Bonferroni correction for multiple comparisons was used for between-group difference in FA, short-range, or long-range FCD measures. Specifically, a $P<0.001$ was used to assess between-group difference in FA measures (i.e., $0.05 / 48$ tests), and a $P<0.0012$ was used to assess between-group difference in short- or long-range FCD measures (i.e., 0.05/42 tests). For partial correlations between FA values and short- or long-range FCD measures, any correlation with an uncorrected $P<0.001$ was reported and was further corrected by false discovery rate (FDR) method for multiple correlation tests (i.e., 48 white matter tracts multiplied by 42 functional hubs).

\section{RESULTS}

\section{The Association of Age with Short- and Long-Range Functional Connectivity Density}

Figure 2 shows the regions with significant between-agegroup differences in short- and long-range FCD mapping. Compared with younger adults, older subjects exhibited a significant reduction in long-range FCD in the lingual, calcarine, postcentral, and precentral cortex, and a significant increase in long-range FCD in the cerebellum, hippocampus, thalamus, insula, caudate, amygdala, fusiform, and anterior cingulate cortex. However, older subjects exhibited a significant reduction in short-range FCD in the lingual, calcarine, postcentral and precentral gyrus, and a significant increase in short-range FCD in the cerebellum, precuneus, posterior cingulate, hippocampus, thalamus, insula, angular gyrus, and supplementary motor area, compared with younger adults (only qualitative results were reported because of large clusters).

Table 2 presents the between-age-group difference in FCD based on 42 functional hub regions. The results are similar to the voxel-wise analysis presented in Figure 2, except that no hub region was found to have significantly reduced FCD in older adults compared with younger people. Older people had increased long-range FCD in hubs of the thalamus $(t=-6.0)$, left and right hippocampus ( $t=-4.7$ and -3.9 , respectively), left fusiform gyrus ( $t=-5.2$ and -3.6 , respectively), and right cerebellum $(t=-3.5)$, as well as increased short-range
FCD in hubs of the left precuneus $(t=-3.3)$, left posterior cingulate $(t=-3.8)$, left inferior parietal $(t=-4.1)$, right angular $(t=-3.7)$, left hippocampus $(t=-3.4)$, left fusiform $(t=-4.0)$, left middle cingulate $(t=-3.2)$, and inferior temporal cortex $(t=-3.5$; all $p<0.001)$.

\section{The Association of Age with White Matter Integrity}

Older people showed a widespread reduction in white matter integrity measured according to FA value, compared with younger adults (Figure 2 and Table 3 ). The most affected region was the column and body of the fornix $(t=13.5)$, followed by the genu of the corpus callosum $(t=9.9)$, anterior and superior corona radiate (both $t=9.6$ ), left and right fornix cres stria terminalis ( $t=9.4$ and 9.2, respectively), tapetum $(t=9.2)$, and body of the corpus callosum $(t=9.2)$. The unaffected white matter tracts were the corticospinal tract, medial lemniscus, part of the cerebellum peduncle, and the cingulum involved in the hippocampus and cingulate gyrus.

\section{Relationship between Functional Connectivity and White Matter Integrity}

Figure 3 and Table 4 show the significant correlation pairs between the FA value of white matter tracts and the FCD of functional hubs. Long-range FCD was generally correlated to the structural integrity of nonadjacent white matter tracts, whereas short-range FCD was correlated to the structural integrity of adjacent white matter tracts. In younger adults, long-range FCD in the right inferior frontal cortex was correlated negatively to the left posterior thalamic radiation $(r=-0.29)$, and short-range FCD in the right angular gyrus was correlated positively to the right posterior corona radiata $(r=0.29)$.

In older adults, long-range FCD in the left precuneus was correlated negatively to wide ranges of white matter tracts, including the splenium of the corpus callosum $(r=-0.35)$, right and left cerebral peduncle ( $r=-0.34$ and -0.35 , respectively), right and left anterior limb of the internal capsule $(r=-0.32$ and -0.33 , respectively), right and left posterior limb of the internal capsule ( $r=-0.37$ and -0.36 , respectively), superior and posterior corona radiata $(r=-0.38$ and -0.40 , respectively), right cingulate gyrus $(r=-0.33)$, and right superior longitudinal fasciculus $(r=-0.38)$, whereas short-range FCD in the left precuneus was correlated positively to the right posterior coronal radiata $(r=-0.32$, all uncorrected $p<0.001)$. After FDR correction, long-range FCD in left precuneus was still significantly correlated with FA values in right posterior corona radiata, right superior corona radiata, and right superior longitudinal fasciculus (Figure 4).

\section{Relationship between Cognitive Function and Neuroimaging Measures}

Cognitive measures (MMSE, digit forward and backward) were not significantly correlated to FCD measures in the younger group. For older adults, the digit span forward test was significantly positively correlated to long-range FCD in the left precuneus $(r=0.33)$ and short-range FCD in the left 


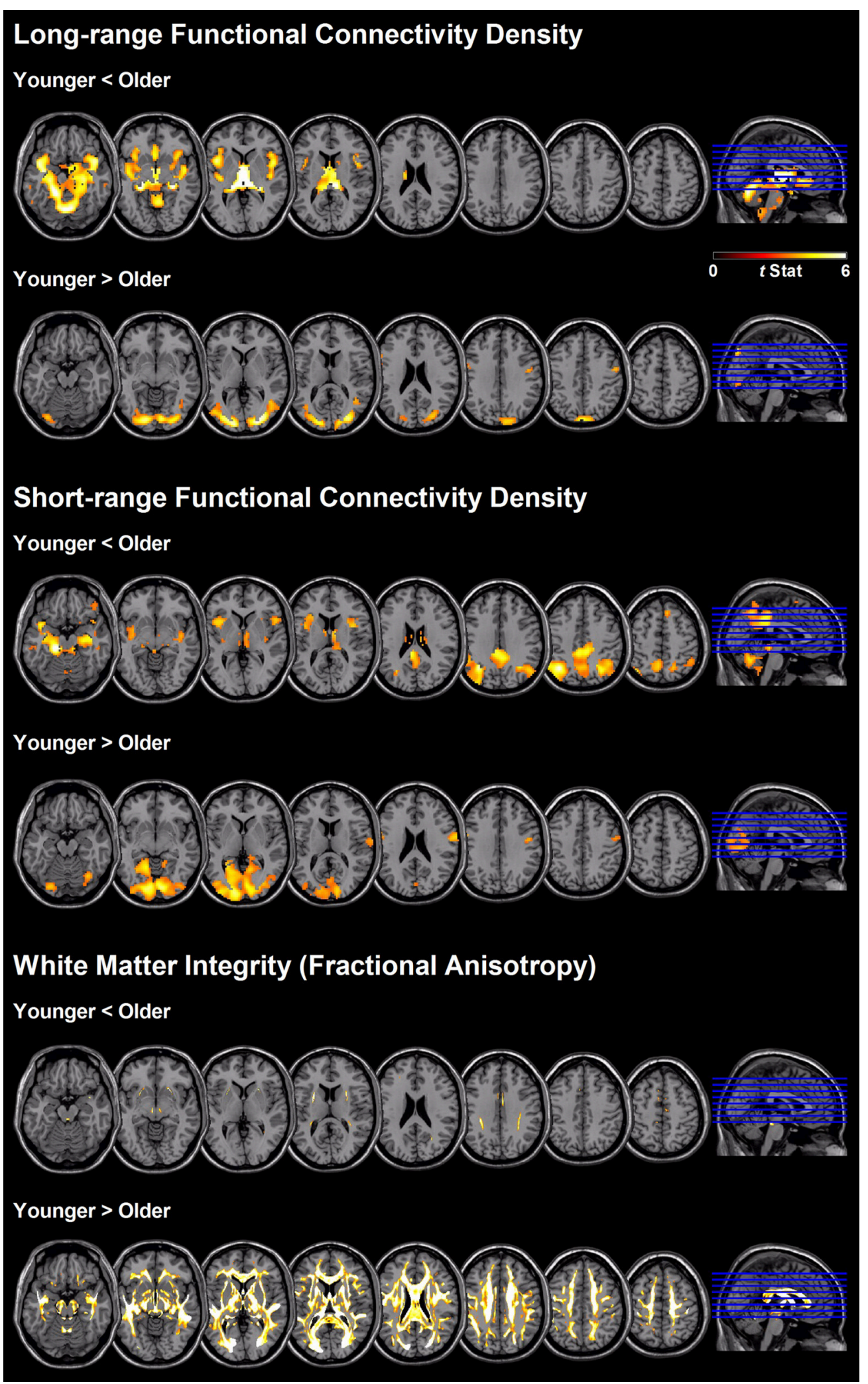

FIGURE 2 | Regional differences between age-groups in short- and long-range FCD, as well as white matter integrity based on fractional anisotropy value. 
TABLE 2 | Between-group difference in long-range and short-range functional connectivity density in $\mathbf{4 2}$ functional hubs.

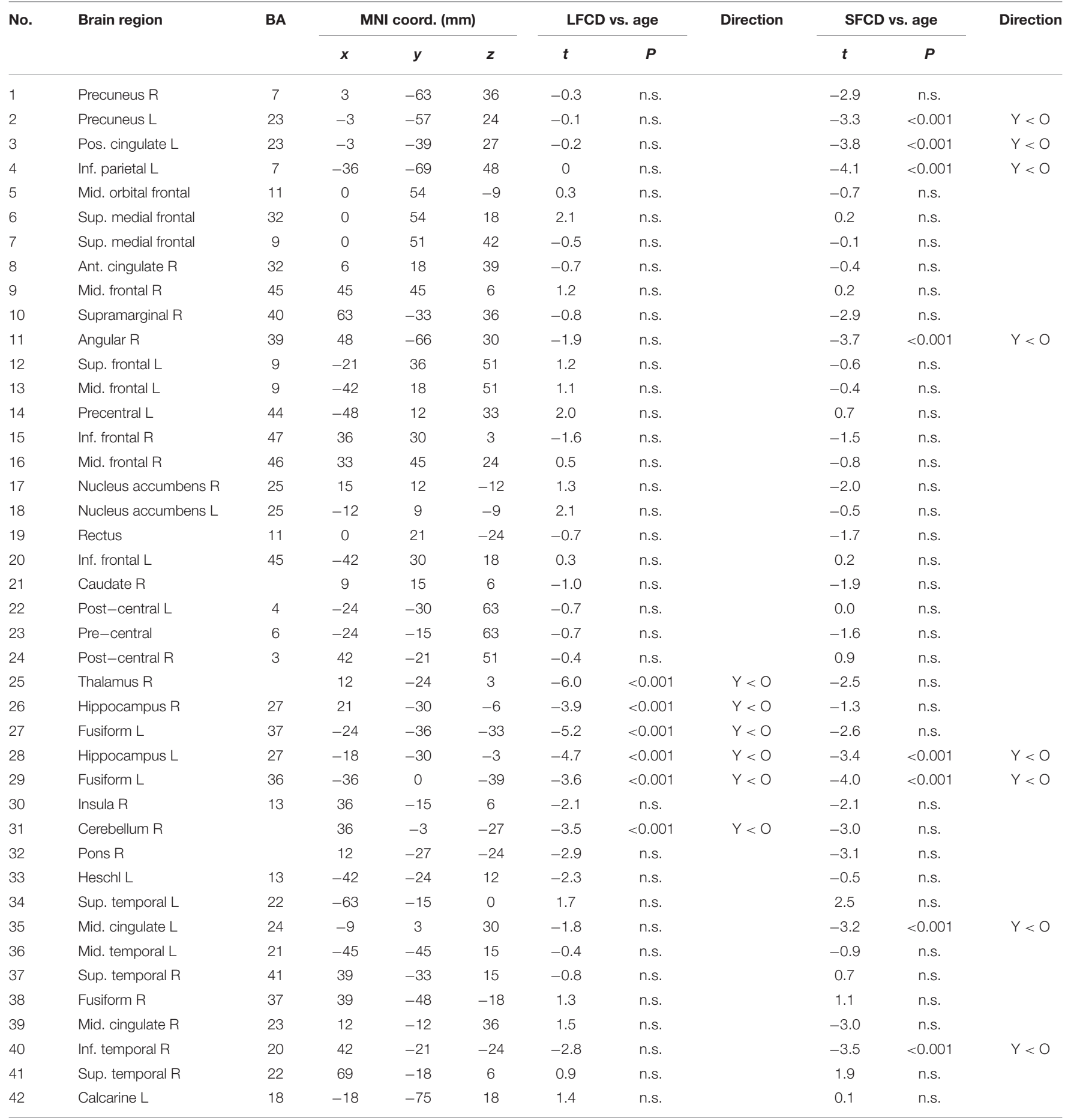

n.s., non-significant. All results had $P<0.0012$ using Bonferroni corrections for multiple comparisons (i.e., 0.05 divided by 42 tests for LFCD or SFCD).

hippocampus $(r=0.36)$. MMSE was positively correlated to long-range FCD in the right angular gyrus $(r=0.30)$, and negatively correlated to the short-range FCD in the right postcentral gyrus $(r=-0.33$, all $p<0.001)$. No significant correlation was found between cognitive measures and white matter integrity in both groups.

\section{DISCUSSION}

Using a combined DTI and resting-state fMRI modalities, we investigated the association of aging with white matter integrity and resting-state functional connectivity, and their relationship in functional connectivity hub regions. Compared 
TABLE 3 | Between-group difference in fractional anisotropy in 48 white matter tracts.

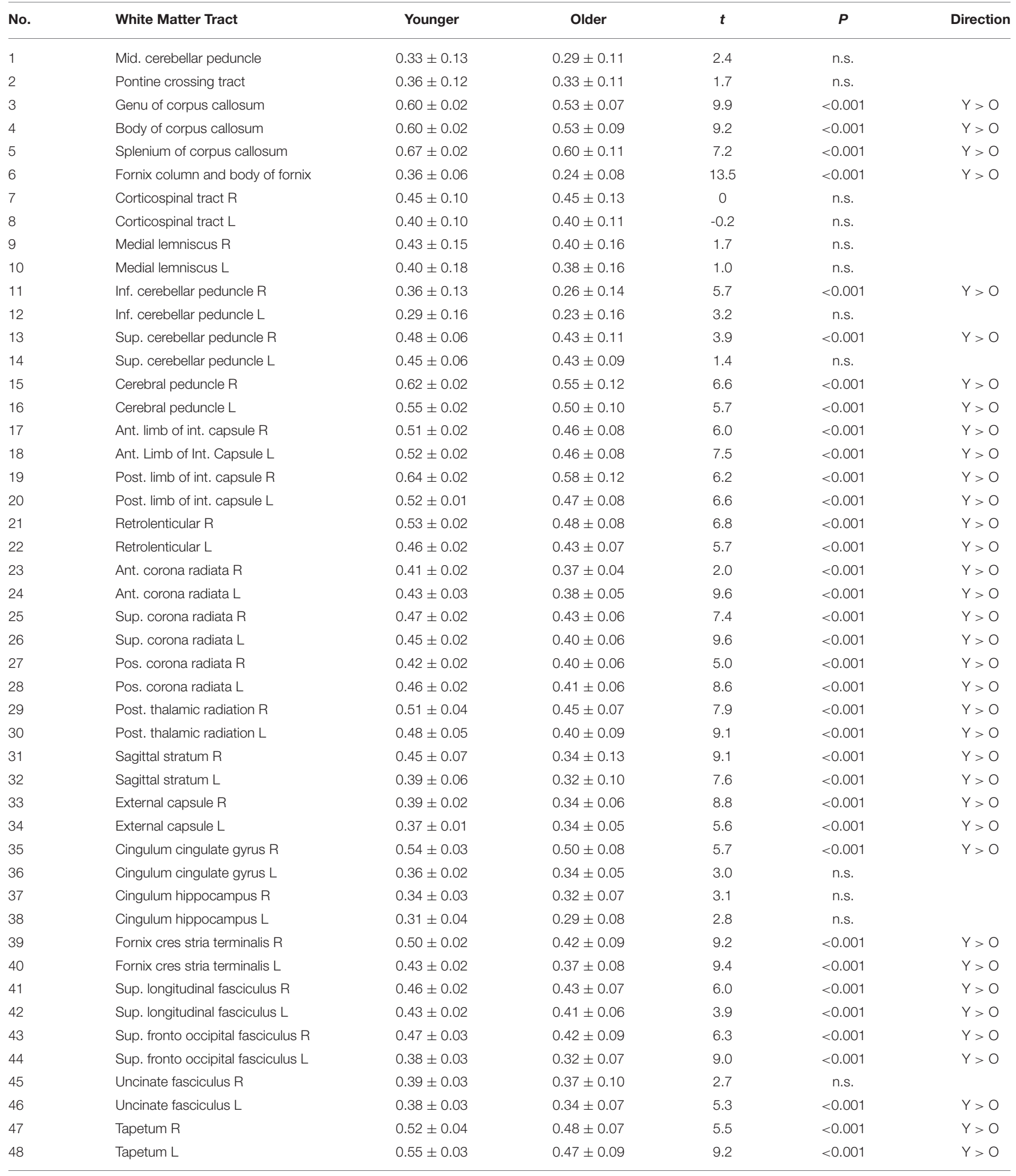

n.s., non-significant. All results had $P<0.001$ using Bonferroni corrections for multiple comparisons (i.e., 0.05 divided by 48 tests). 


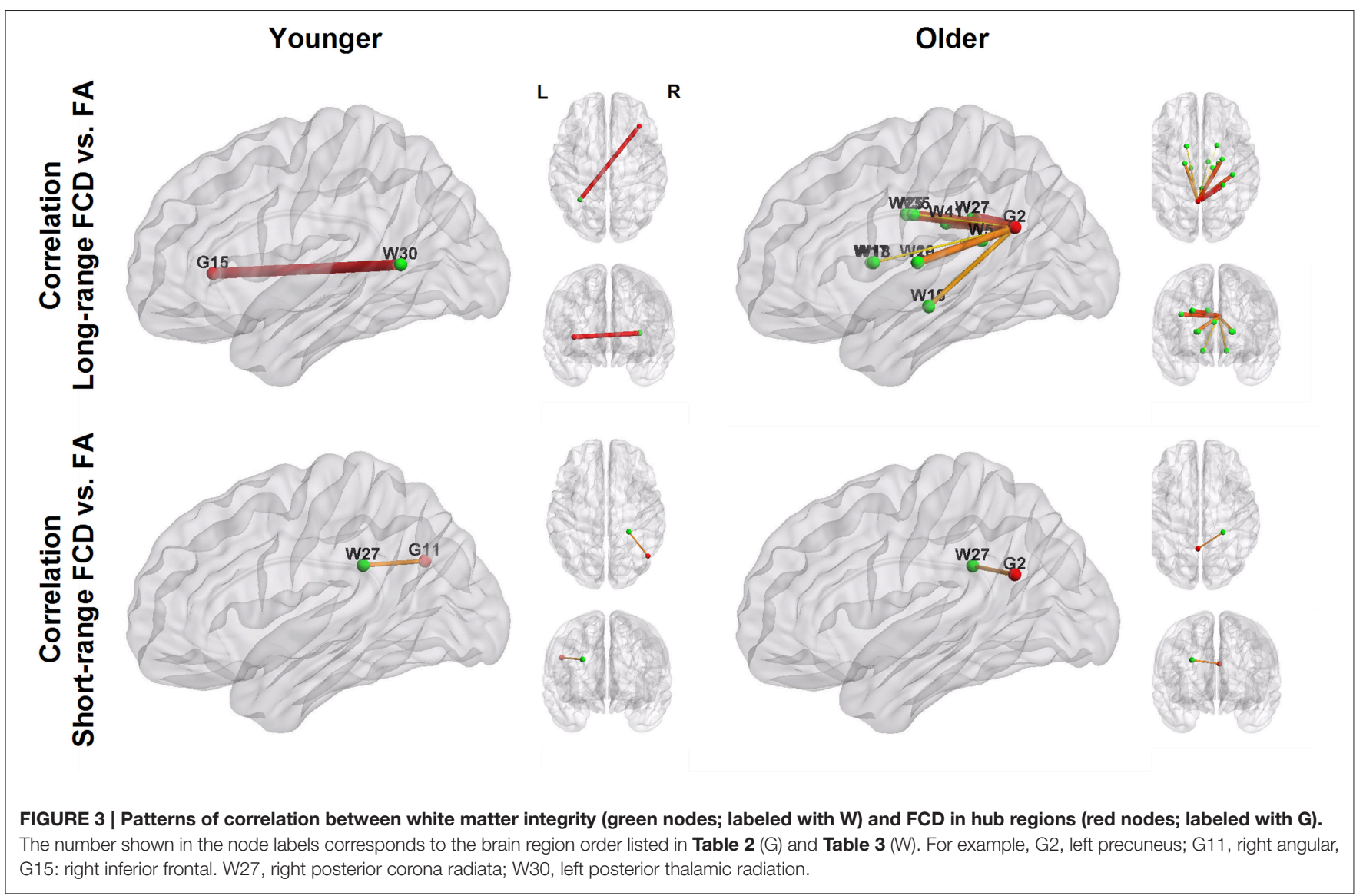

with the younger group, older adults showed more widespread reductions in white matter integrity, with selective preservation in brain stem tracts and the cingulum connected to the hippocampus and cingulate cortex, whereas short- and longrange FCD exhibited reduced FCD in the occipital, postcentral, and precentral cortex and increased FCD in DMN regions. In functional hub regions, older adults exhibited significantly increased FCD in the precuneus, posterior and middle cingulate, thalamus, hippocampus, fusiform gyrus, and inferior temporal cortex. Furthermore, the associations between white matter integrity and FCD in the functional hubs were identified primarily in older adults where short- and long-range FCD in the left precuneus was negatively correlated to the structural integrity of adjacent and nonadjacent white matter tracts, respectively. We also found that long-range FCD in the left precuneus was positively correlated to cognitive function. These findings suggest that the precuneus plays a crucial role in normal aging in the DTI-fMRI relationship and support the compensatory hypothesis of neurocognitive aging theory.

\section{Association of Age with White Matter Integrity}

Our findings of widespread reduced white matter integrity in older adults compared with younger adults are consistent with prior DTI reports (Pfefferbaum et al., 2000; Salat et al., 2005; Pagani et al., 2008; Heise et al., 2011). In addition, our observations of uneven age-associated decline in white matter integrity are consistent with prior reports (Pagani et al., 2008; Heise et al., 2011) that older adults had significant reductions in white matter integrity in the fornix, corpus callosum, and corona radiata. These affected white matter tracts involved projection, association, and commission fibers in the brain. Furthermore, we found that white matter integrity in the brain stem, cerebellum, and cingulum connected to the hippocampus and cingulate gyrus was relatively preserved in older adults. These preserved white matter regions were brainstem tracts and parts of association fibers (cingulum).

Our results suggest that normal aging is less associated with the integrity of white matter tracts that are related to vital body function, and intriguingly, the selective preservation of the cingulum in our healthy cohort may elucidate the differential effects of normal aging and neurodegenerative disease, such as Alzheimer's disease. The cingulum has been invariantly implicated in neurodegenerative disease (Rose et al., 2000; Zhang et al., 2007). The preservation of cingulum integrity in older adults and the absence of correlation between cognitive function and white matter integrity found in this study suggest that cognitive decline in normal aging is not driven by structural changes. 
TABLE 4 | Significant correlations of white matter integrity with functional connectivity density in hub regions.

\begin{tabular}{|c|c|c|c|c|}
\hline Gray Matter Hub & White Matter Region & Correlation $r$ & $P$ uncorrected & P FDR-corrected \\
\hline \multicolumn{5}{|l|}{ YOUNGER } \\
\hline \multicolumn{5}{|c|}{ Long-range Functional Connectivity Density } \\
\hline Inferior frontal R & Posterior thalamic radiation $\mathrm{L}$ & -0.29 & 0.001 & \\
\hline \multicolumn{5}{|c|}{ Short-range Functional Connectivity Density } \\
\hline Angular R & Posterior corona radiata $\mathrm{R}$ & 0.29 & 0.001 & \\
\hline \multicolumn{5}{|l|}{ OLDER } \\
\hline \multicolumn{5}{|c|}{ Long-range Functional Connectivity Density } \\
\hline Precuneus L & Splenium of corpus callosum & -0.35 & $<0.001$ & \\
\hline Precuneus L & Cerebral peduncle R & -0.34 & $<0.001$ & \\
\hline Precuneus L & Cerebral peduncle $L$ & -0.35 & $<0.001$ & \\
\hline Precuneus L & Anterior limb of internal capsule R & -0.32 & $<0.001$ & \\
\hline Precuneus L & Anterior limb of internal capsule $L$ & -0.33 & $<0.001$ & \\
\hline Precuneus L & Posterior limb of internal capsule R & -0.37 & $<0.001$ & \\
\hline Precuneus L & Posterior limb of internal capsule $L$ & -0.36 & $<0.001$ & \\
\hline Precuneus L & Superior corona radiata $\mathrm{R}$ & -0.38 & $<0.001$ & $<0.05$ \\
\hline Precuneus L & Posterior corona radiata $\mathrm{R}$ & -0.40 & $<0.001$ & $<0.05$ \\
\hline Precuneus L & Cingulum cingulate gyrus $R$ & -0.33 & $<0.001$ & \\
\hline Precuneus L & Superior longitudinal fasciculus $\mathrm{R}$ & -0.38 & $<0.001$ & $<0.05$ \\
\hline \multicolumn{5}{|c|}{ Short-range Functional Connectivity Density } \\
\hline Precuneus L & Posterior corona radiata $\mathrm{R}$ & -0.32 & $<0.001$ & \\
\hline
\end{tabular}

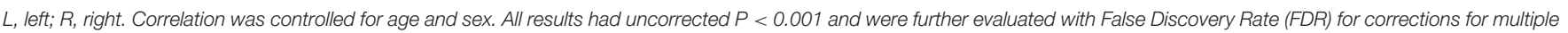
comparisons.

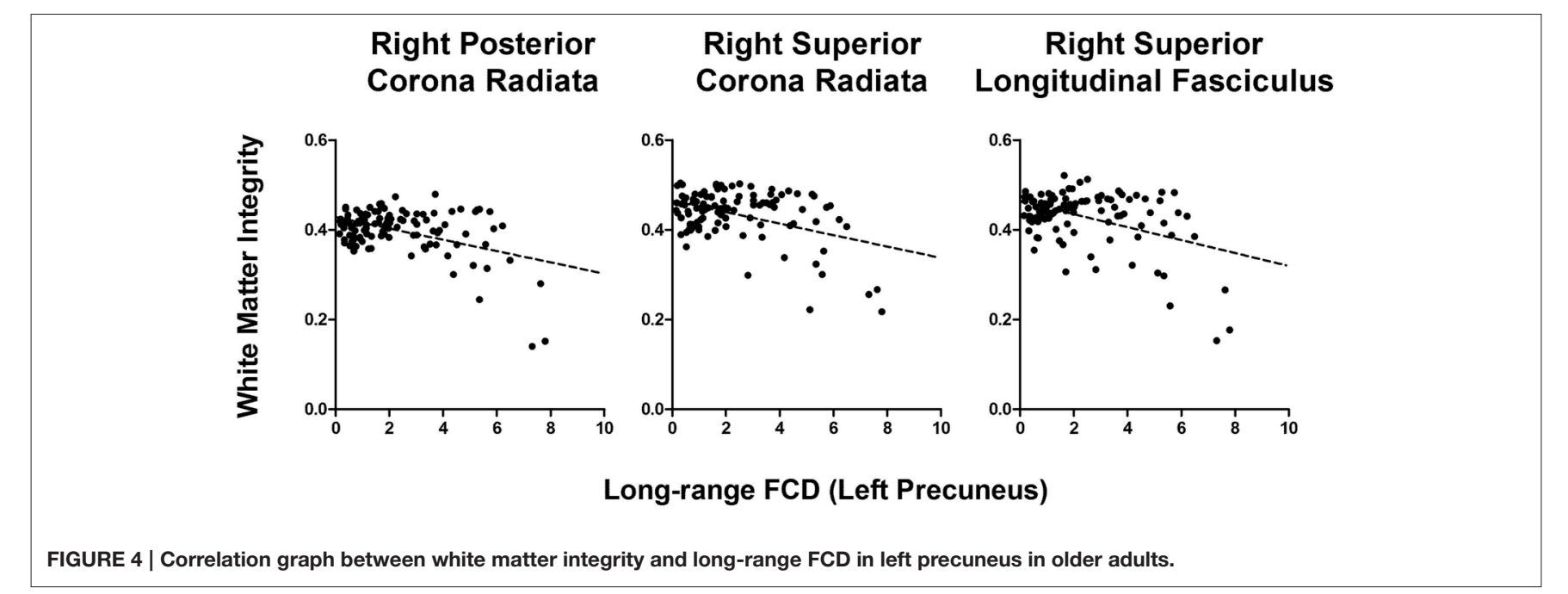

\section{Association of Age with Resting-State Functional Connectivity}

Contrary to prior reports based on a public resting-state fMRI database (Tomasi and Volkow, 2012a), we found a diverse pattern of short- and long-range FCD in younger and older adults. Tomasi et al. reported that long-range FCD was negatively correlated to age in the posterior cingulate and ventral precuneus region, the core of DMN, and both short- and long-range FCD was positively correlated to age in the postcentral gyrus. By contrast, using the same FCD method, we found both increased short- and long-range FCD in DMN, and reduced FCD in the visual, somatosensory, and motor cortex.

The discrepancy in the results may be due to the predominance of younger subjects and the lack of older subjects (aged $>60$ ) in the public database (1000 Functional Connectomes Project), which leads to statistical bias when applying Pearson's correlation to the relationship between age and FCD measures. However, a recent seed-based study revealed that resting-state connectivity in the posterior cingulate was negatively correlated with age in a continuous aging 
cohort (Mevel et al., 2013). Whereas, the reduced resting-state functional connectivity in DMN was the most common pattern reported in the elderly population (see Ferreira and Busatto, 2013 for reviews), numerous studies have revealed that age is positively correlated to functional connectivity in DMN areas (Meunier et al., 2009; Biswal et al., 2010; Toussaint et al., 2011). Furthermore, although the FCD method is capable of detecting DMN hubs and other functional networks, we found that our results of reduced FCD in visual, somatosenroy, and motor networks (except the cerebellum) in older adults differed from those reported by Tomasi and Volkow (2012a). Similarly, the inconsistency of the aging effect on these networks has also been reported previously (Wu et al., 2007; Allen et al., 2011).

Another reason for discrepancy of FCD results might be that our resting-state fMRI data were not pre-processed in the same way with Tomasi and Volkow (2012a). Tomasi and Volkow, for instance, regressed the global signal, which was deliberately not done in the present study to avoid introducing distortions in the time series data (Murphy et al., 2009; Anderson et al., 2011). Moreover, contrast to our single site neuroimaging data, the site effects (i.e., fMRI data collected from different MRI protocols and machines) in 1000 Functional Connectomes Project might also contribute the difference in FCD results.

\section{Association of Cognitive Function with Neuroimaging Measures}

Decline in cognitive performance in old age is linked to both suboptimal neural processing in gray matter (Rajah and D'esposito, 2005) and reduced integrity of white matter (Madden et al., 2012). There have been a number of studies showing altered functional connectivity related to healthy aging and/or dementia, such as Alzheimer's disease, in which the later has been characterized as a connectivity disease (Van Den Heuvel and Hulshoff Pol, 2010; Hafkemeijer et al., 2012; Salami et al., 2014). For example, age-related disruption in cortico-hippocampal functional connectivity could lead to a more functionally isolated hippocampus at rest, suggesting aberrant hippocampal decoupling and deficits during mnemonic processing (Salami et al., 2014).

We did not find the correlation between cognitive function and the integrity of structural connectivity as measured by FA values. However, we did find positive correlations between FCD and digit span tasks/MMSE in precuneus, hippocampus, or angular gyrus in older group, suggesting increased restingstate connectivity in major functional hub is associated with preserved cognitive functions. Conversely, we found a negative correlation between MMSE and FCD in post-central gyrus, a region typically being activated during cognitive tasks and may reflect and anti-correlation between resting-state and salience network.

\section{Implication of the DTI-fMRI Relationship in Normal Aging}

Previous reports (Persson et al., 2006; Madden et al., 2007) have primarily reported DTI-fMRI relationships in older adults, showing negative correlations between FCD and white matter integrity. A recent study shows that increased connectivity in the compensatory network correlates positively with preserved white-matter integrity in bilateral fronto-parietal tracks (Burianova et al., 2015). Another study shows that greater standard deviation (SD) of BOLD signal was associated with better fluid abilities and memory and older adults with greater white matter integrity in all major white matter tracts had also greater SD of BOLD signal and better performance on tests of memory and fluid abilities (Burzynska et al., 2015). Marstaller et al. shows that age-related decline in white matter integrity and gray matter volume is associated with activity in prefrontal nodes of the salience and fronto-parietal network, possibly reflecting compensatory mechanisms (Marstaller et al., 2015). Collectively, these findings suggest the importance of structural integrity and functional connectivity in working memory performance associated with healthy aging.

Several neurocognitive aging theories have been proposed in relation to the DTI-fMRI relationship, including compensation, neural efficiency, nonselective overrecruitment, or underrecruitment (Bennett and Rypma, 2013). Our results supported compensation theory, that while normal aging was strongly associated with reduced structural integrity, the preservation of white matter tracts in the cingulum and increased FCD in DMN areas may help to compensate for the aging-related structural impairment and maintain healthy congitive function.

Compensatory increases in functional connectivity may be triggered to recruit additional neuronal resources when available structural networks, such as white matter integrity, are diminished (Reuter-Lorenz and Cappell, 2008; Park and Reuter-Lorenz, 2009; Cabeza and Dennis, 2012). This notion can be further supported by our results that older adults showed a positive correlation between cognitive assessments and FCD in DMN regions, and the predominantly negative correlations between FCD in the left precuneus and white matter intergrity. We did not find a significant DTI-cognitive or fMRI-cognitive correlation in younger people, and identified only two DTIfMRI associations (Table 4). Thus our results support neither compensatory nor other neurocognitive mechanisms in younger people.

The structural integrity is expected to influence neural activity in the connected gray matter areas (i.e., at the terminals of the white matter tracts) compared to other gray matter regions to which they are not connected or only indirectly connected (Logothetis et al., 2001; Bennett and Rypma, 2013). Prior studies show that younger adult has positive correlation between white matter integrity and fMRI activation in adjacent gray matter regions (Toosy et al., 2004; Forstmann et al., 2008; Kim and Whalen, 2009; Van Eimeren et al., 2010) or partially adjacent gray matter region (Koch et al., 2010). In contrast, DTI-fMRI correlations were mostly negative for studies examining white matter integrity and fMRI activation in non-adjacent brain regions (Baird et al., 2005; Persson et al., 2006; Madden et al., 2007; Putnam et al., 2008; De Chastelaine et al., 2011).

Structural connectivity can influence the strength of functional connectivity (Damoiseaux and Greicius, 2009; Honey et al., 2010). Therefore, short- or long-range FCD is likely to be influenced by the integrity of white matter tracts. Indeed, 
we found that white matter integrity was correlated negatively with long-range FCD mostly in older people, and the DTI-fMRI relationship was associated with spatial proximity between the functional hubs and white matter tracts (Figure 3). When considering the negative correlation of DTI-fMRI in older adults, the association between the range of FCD and spatial proximity of white matter tracts further strengthens the compensatory hypothesis and helps to clarify the inconsistent findings of previous studies.

\section{LIMITATIONS}

This study was subject to certain limitations. First, we examined neither the effect of sex, nor the interaction effect of sex and age on the between-group difference of neuroimaging measures, because the sex effect was not the primary focus of this study. However, we did control the effects of age and sex on FCD-FA correlations. Prior studies show that female participants exhibit greater overall cortical connectivity and more efficient local and global cortical networks than male subjects (Gong et al., 2009, 2011; Tomasi and Volkow, 2012b). The association of gender with functional connectivity and structural integrity is warranted to be investigated in the future based on a larger aging sample. Second, the FCD method quantified only the density of functional connections, but discarded the spatial connectivity patterns. However, when studying the DTI-fMRI relationship, this approach can greatly simplify the dimension of complex brain networks and provide a broader view of DTI-fMRI relationship. Third, the ROI approach may lower the strength of the DTI-fMRI correlation (Table 4; $r$ square 10-16\%). Nevertheless, our results provide a starting point for investigating the detailed DTI-fMRI relationship using specific seed-based analyses. We note that the DTIfMRI relationship may be nonlinear (Figure 4), which warrants additional study to model the DTI-fMRI relationship using nonlinear regression. Finally, the BOLD signal is an indirect measure of neural activity and is heavily influenced by the efficiency of neurovascular coupling. Aging may be associated with changes in cerebrovascular dynamics, which confounds

\section{REFERENCES}

Allen, E. A., Erhardt, E. B., Damaraju, E., Gruner, W., Segall, J. M., Silva, R. F., et al. (2011). A baseline for the multivariate comparison of resting-state networks. Front. Syst. Neurosci. 5:2. doi: 10.3389/fnsys.2011.00002

Anderson, J. S., Druzgal, T. J., Lopez-Larson, M., Jeong, E. K., Desai, K., and Yurgelun-Todd, D. (2011). Network anticorrelations, global regression, and phase-shifted soft tissue correction. Hum. Brain Mapp. 32, 919-934. doi: 10.1002/hbm. 21079

Baird, A. A., Colvin, M. K., Vanhorn, J. D., Inati, S., and Gazzaniga, M. S. (2005). Functional connectivity: integrating behavioral, diffusion tensor imaging, and functional magnetic resonance imaging data sets. J. Cogn. Neurosci. 17, 687-693. doi: 10.1162/0898929053467569

Bennett, I. J., and Rypma, B. (2013). Advances in functional neuroanatomy: a review of combined DTI and fMRI studies in healthy younger and older adults. Neurosci. Biobehav. Rev. 37, 1201-1210. doi: 10.1016/j.neubiorev.2013. 04.008 the FCD analyses (Ferreira and Busatto, 2013). Furthermore, recent evidence has suggested that increased neural activity is not always associated with changes in cerebral blood flows (Huo et al., 2014); thus, our findings of DTI-fMRI association may overlook certain brain regions, such as the frontal cortex.

\section{CONCLUSIONS}

This study comprehensively investigated the association of aging with the DTI-fMRI relationship by using a model-free analytic approach (i.e., FCD mapping and whole-brain white matter segmentation). Although normal aging was associated with widespread decline in white matter integrity and reduced visual, somatosensory, and motor functional connectivity, increased FCD in the DMN hub of the precuneus was associated with reduced white matter integrity and may be linked to the compensation of cognitive capacity in healthy elderly adults against the aging process.

\section{AUTHOR CONTRIBUTIONS}

AY designed the study, analyzed image data, performed statistical results, and drafted the manuscript. $\mathrm{ML}$ and $\mathrm{CH}$ performed the experiment and collected the data. ST and CL designed the study and gave critical comments on the manuscript.

\section{ACKNOWLEDGMENTS}

This work was supported by Taipei Veterans General Hospital, Taiwan (grants VGHUST102-G1-2-1, and VGHUST103-G14-1); Taipei Veterans General Hospital-National Yang-Ming University-Excellent Physician Scientists Cultivation Program, No. 103-V-A-002, and the Ministry of Science and Technology (MOST) of Taiwan (grant MOST 103-2314-B-075 -067 MY3; MOST 104-2314-B-075 -072 -MY3; MOST 104-2314-B075 -078 -MY2); and the MOST support for the Center for Dynamical Biomarkers and Translational Medicine, National Central University, Taiwan (grant MOST 104-2911-I-008-001).

Biswal, B. B., Mennes, M., Zuo, X. N., Gohel, S., Kelly, C., Smith, S. M., et al. (2010). Toward discovery science of human brain function. Proc. Natl. Acad. Sci. U.S.A. 107, 4734-4739. doi: 10.1073/pnas.0911855107

Boorman, E. D., O'shea, J., Sebastian, C., Rushworth, M. F., and JohansenBerg, H. (2007). Individual differences in white-matter microstructure reflect variation in functional connectivity during choice. Curr. Biol. 17, 1426-1431. doi: 10.1016/j.cub.2007.07.040

Burianova, H., Marstaller, L., Choupan, J., Sepehrband, F., Ziaei, M., and Reutens, D. (2015). The relation of structural integrity and task-related functional connectivity in the aging brain. Neurobiol. Aging 36, 2830-2837. doi: 10.1016/j.neurobiolaging.2015.07.006

Burzynska, A. Z., Wong, C. N., Voss, M. W., Cooke, G. E., McAuley, E., and Kramer, A. F. (2015). White matter integrity supports BOLD signal variability and cognitive performance in the aging human brain. PLoS ONE 10:e0120315. doi: 10.1371/journal.pone.0120315

Cabeza, R., and Dennis, N. A. (2012). Frontal Lobes and Aging: Deterioration and Compensation. New York, NY: Oxford University. 
Damoiseaux, J. S., and Greicius, M. D. (2009). Greater than the sum of its parts: a review of studies combining structural connectivity and resting-state functional connectivity. Brain Struct. Funct. 213, 525-533. doi: 10.1007/s00429-0090208-6

De Chastelaine, M., Wang, T. H., Minton, B., Muftuler, L. T., and Rugg, M. D. (2011). The effects of age, memory performance, and callosal integrity on the neural correlates of successful associative encoding. Cereb. Cortex 21, 2166-2176. doi: 10.1093/cercor/bhq294

Ferreira, L. K., and Busatto, G. F. (2013). Resting-state functional connectivity in normal brain aging. Neurosci. Biobehav. Rev. 37, 384-400. doi: 10.1016/j.neubiorev.2013.01.017

Folstein, M. F., Folstein, S. E., and McHugh, P. R. (1975). "Mini-mental state”. A practical method for grading the cognitive state of patients for the clinician. J. Psychiatr. Res. 12, 189-198. doi: 10.1016/0022-3956(75)90026-6

Forstmann, B. U., Jahfari, S., Scholte, H. S., Wolfensteller, U., Van Den Wildenberg, W. P., and Ridderinkhof, K. R. (2008). Function and structure of the right inferior frontal cortex predict individual differences in response inhibition: a model-based approach. J. Neurosci. 28, 9790-9796. doi: 10.1523/JNEUROSCI.1465-08.2008

Fox, M. D., and Raichle, M. E. (2007). Spontaneous fluctuations in brain activity observed with functional magnetic resonance imaging. Nat. Rev. Neurosci. 8, 700-711. doi: 10.1038/nrn2201

Gong, G., He, Y., and Evans, A. C. (2011). Brain connectivity: gender makes a difference. Neuroscientist 17, 575-591. doi: 10.1177/1073858410386492

Gong, G., Rosa-Neto, P., Carbonell, F., Chen, Z. J., He, Y., and Evans, A. C. (2009). Age- and gender-related differences in the cortical anatomical network. J. Neurosci. 29, 15684-15693. doi: 10.1523/JNEUROSCI.2308-09.2009

Good, C. D., Johnsrude, I. S., Ashburner, J., Henson, R. N., Friston, K. J., and Frackowiak, R. S. (2001). A voxel-based morphometric study of ageing in 465 normal adult human brains. Neuroimage 14, 21-36. doi: 10.1006/nimg.2001.0786

Greicius, M. D., Supekar, K., Menon, V., and Dougherty, R. F. (2009). Restingstate functional connectivity reflects structural connectivity in the default mode network. Cereb. Cortex 19, 72-78. doi: 10.1093/cercor/bhn059

Hafkemeijer, A., Van Der Grond, J., and Rombouts, S. A. (2012). Imaging the default mode network in aging and dementia. Biochim. Biophys. Acta 1822, 431-441. doi: 10.1016/j.bbadis.2011.07.008

Heise, V., Filippini, N., Ebmeier, K. P., and MacKay, C. E. (2011). The APOE varepsilon 4 allele modulates brain white matter integrity in healthy adults. Mol. Psychiatry 16, 908-916. doi: 10.1038/mp.2010.90

Honey, C. J., Thivierge, J. P., and Sporns, O. (2010). Can structure predict function in the human brain? Neuroimage 52, 766-776. doi: 10.1016/j.neuroimage.2010.01.071

Hughes, C. P., Berg, L., Danziger, W. L., Coben, L. A., and Martin, R. L. (1982). A new clinical scale for the staging of dementia. Br. J. Psychiatry 140, 566-572. doi: 10.1192/bjp.140.6.566

Huo, B. X., Smith, J. B., and Drew, P. J. (2014). Neurovascular coupling and decoupling in the cortex during voluntary locomotion. J. Neurosci. 34, 10975-10981. doi: 10.1523/JNEUROSCI.1369-14.2014

Kim, M. J., and Whalen, P. J. (2009). The structural integrity of an amygdalaprefrontal pathway predicts trait anxiety. J. Neurosci. 29, 11614-11618. doi: 10.1523/JNEUROSCI.2335-09.2009

Koch, K., Wagner, G., Dahnke, R., Schachtzabel, C., Gullmar, D., Reichenbach, J. R., et al. (2010). Structure-function relationships in the context of reinforcement-related learning: a combined diffusion tensor imagingfunctional magnetic resonance imaging study. Neuroscience 168, 190-199. doi: 10.1016/j.neuroscience.2010.03.026

Koch, M. A., Norris, D. G., and Hund-Georgiadis, M. (2002). An investigation of functional and anatomical connectivity using magnetic resonance imaging. Neuroimage 16, 241-250. doi: 10.1006/nimg.2001.1052

Logothetis, N. K., Pauls, J., Augath, M., Trinath, T., and Oeltermann, A. (2001). Neurophysiological investigation of the basis of the fMRI signal. Nature 412, 150-157. doi: 10.1038/35084005

Madden, D. J., Bennett, I. J., Burzynska, A., Potter, G. G., Chen, N. K., and Song, A. W. (2012). Diffusion tensor imaging of cerebral white matter integrity in cognitive aging. Biochim. Biophys. Acta 1822, 386-400. doi: 10.1016/j.bbadis.2011.08.003
Madden, D. J., Spaniol, J., Whiting, W. L., Bucur, B., Provenzale, J. M., Cabeza, R., et al. (2007). Adult age differences in the functional neuroanatomy of visual attention: a combined fMRI and DTI study. Neurobiol. Aging 28, 459-476. doi: 10.1016/j.neurobiolaging.2006.01.005

Marstaller, L., Williams, M., Rich, A., Savage, G., and Burianova, H. (2015). Aging and large-scale functional networks: white matter integrity, gray matter volume, and functional connectivity in the resting state. Neuroscience 290, 369-378. doi: 10.1016/j.neuroscience.2015.01.049

Meunier, D., Achard, S., Morcom, A., and Bullmore, E. (2009). Age-related changes in modular organization of human brain functional networks. Neuroimage 44, 715-723. doi: 10.1016/j.neuroimage.2008.09.062

Mevel, K., Landeau, B., Fouquet, M., La Joie, R., Villain, N., Mezenge, F., et al. (2013). Age effect on the default mode network, inner thoughts, and cognitive abilities. Neurobiol. Aging 34, 1292-1301. doi: 10.1016/j.neurobiolaging.2012.08.018

Minati, L., Grisoli, M., and Bruzzone, M. G. (2007). MR spectroscopy, functional MRI, and diffusion-tensor imaging in the aging brain: a conceptual review. J. Geriatr. Psychiatry Neurol. 20, 3-21. doi: 10.1177/0891988706297089

Morrison, J. H., and Hof, P. R. (1997). Life and death of neurons in the aging brain. Science 278, 412-419. doi: 10.1126/science.278.5337.412

Murphy, K., Birn, R. M., Handwerker, D. A., Jones, T. B., and Bandettini, P. A. (2009). The impact of global signal regression on resting state correlations: are anti-correlated networks introduced? Neuroimage 44, 893-905. doi: 10.1016/j.neuroimage.2008.09.036

Pagani, E., Agosta, F., Rocca, M. A., Caputo, D., and Filippi, M. (2008). Voxel-based analysis derived from fractional anisotropy images of white matter volume changes with aging. Neuroimage 41, 657-667. doi: 10.1016/j.neuroimage.2008.03.021

Park, D. C., and Reuter-Lorenz, P. (2009). The adaptive brain: aging and neurocognitive scaffolding. Annu. Rev. Psychol. 60, 173-196. doi: 10.1146/annurev.psych.59.103006.093656

Persson, J., Nyberg, L., Lind, J., Larsson, A., Nilsson, L. G., Ingvar, M., et al. (2006). Structure-function correlates of cognitive decline in aging. Cereb. Cortex 16, 907-915. doi: 10.1093/cercor/bhj036

Pfefferbaum, A., Sullivan, E. V., Hedehus, M., Lim, K. O., Adalsteinsson, E., and Moseley, M. (2000). Age-related decline in brain white matter anisotropy measured with spatially corrected echo-planar diffusion tensor imaging. Magn. Reson. Med. 44, 259-268. doi: 10.1002/1522-2594(200008)44:2\&lt;259::AIDMRM13\&gt;3.0.CO;2-6

Putnam, M. C., Wig, G. S., Grafton, S. T., Kelley, W. M., and Gazzaniga, M. S. (2008). Structural organization of the corpus callosum predicts the extent and impact of cortical activity in the nondominant hemisphere. J. Neurosci. 28, 2912-2918. doi: 10.1523/JNEUROSCI.2295-07.2008

Rajah, M. N., and D'esposito, M. (2005). Region-specific changes in prefrontal function with age: a review of PET and fMRI studies on working and episodic memory. Brain 128, 1964-1983. doi: 10.1093/brain/awh608

Raz, N., Rodrigue, K. M., and Haacke, E. M. (2007). Brain aging and its modifiers: insights from in vivo neuromorphometry and susceptibility weighted imaging. Ann. N.Y. Acad. Sci. 1097, 84-93. doi: 10.1196/annals.1379.018

Reuter-Lorenz, P. A., and Cappell, K. A. (2008). Neurocognitive Aging and the Compensation Hypothesis. Curr. Dir. Psychol. Sci. 17, 177-182. doi: 10.1111/j.1467-8721.2008.00570.x

Rose, S. E., Chen, F., Chalk, J. B., Zelaya, F. O., Strugnell, W. E., Benson, M., et al. (2000). Loss of connectivity in Alzheimer's disease: an evaluation of white matter tract integrity with colour coded MR diffusion tensor imaging. J. Neurol. Neurosurg. Psychiatr. 69, 528-530. doi: 10.1136/jnnp.69.4.528

Salami, A., Pudas, S., and Nyberg, L. (2014). Elevated hippocampal resting-state connectivity underlies deficient neurocognitive function in aging. Proc. Natl. Acad. Sci. U.S.A. 111, 17654-17659. doi: 10.1073/pnas.1410233111

Salat, D. H., Tuch, D. S., Greve, D. N., Van Der Kouwe, A. J., Hevelone, N. D., Zaleta, A. K., et al. (2005). Age-related alterations in white matter microstructure measured by diffusion tensor imaging. Neurobiol. Aging 26, 1215-1227. doi: 10.1016/j.neurobiolaging.2004.09.017

Scahill, R. I., Frost, C., Jenkins, R., Whitwell, J. L., Rossor, M. N., and Fox, N. C. (2003). A longitudinal study of brain volume changes in normal aging using serial registered magnetic resonance imaging. Arch. Neurol. 60, 989-994. doi: 10.1001/archneur.60.7.989 
Sheehan, D. V., Lecrubier, Y., Sheehan, K. H., Amorim, P., Janavs, J., Weiller, E., et al. (1998). The Mini-International Neuropsychiatric Interview (M.I.N.I.): the development and validation of a structured diagnostic psychiatric interview for DSM-IV and ICD-10. J. Clin. Psychiatry 59(Suppl. 20), 22-33.

Tomasi, D., and Volkow, N. D. (2010). Functional connectivity density mapping. Proc. Natl. Acad. Sci. U.S.A. 107, 9885-9890. doi: 10.1073/pnas.1001414107

Tomasi, D., and Volkow, N. D. (2011). Association between functional connectivity hubs and brain networks. Cereb. Cortex 21, 2003-2013. doi: 10.1093/cercor/bhq268

Tomasi, D., and Volkow, N. D. (2012a). Aging and functional brain networks. Mol. Psychiatry 17, 549-558. doi: 10.1038/mp.2011.81

Tomasi, D., and Volkow, N. D. (2012b). Laterality patterns of brain functional connectivity: gender effects. Cereb. Cortex 22, 1455-1462. doi: $10.1093 /$ cercor/bhr230

Toosy, A. T., Ciccarelli, O., Parker, G. J., Wheeler-Kingshott, C. A., Miller, D. H., and Thompson, A. J. (2004). Characterizing function-structure relationships in the human visual system with functional MRI and diffusion tensor imaging. Neuroimage 21, 1452-1463. doi: 10.1016/j.neuroimage.2003.11.022

Toussaint, P. J., Maiz, S., Coynel, D., Messe, A., Perlbarg, V., Habert, M. O., et al. (2011). "Characterization of the default mode functional connectivity in normal aging and Alzheimer's disease: an approach combining entropy-based and graph theoretical measurements," in IEEE International Symposium on Biomedical Imaging: From Nano to Macro (Chicago, IL), 853-856.

Van Den Heuvel, M. P., and Hulshoff Pol, H. E. (2010). Exploring the brain network: a review on resting-state fMRI functional connectivity. Eur. Neuropsychopharmacol. 20, 519-534. doi: 10.1016/j.euroneuro.2010.03.008

Van Eimeren, L., Grabner, R. H., Koschutnig, K., Reishofer, G., Ebner, F., and Ansari, D. (2010). Structure-function relationships underlying calculation: a combined diffusion tensor imaging and fMRI study. Neuroimage 52, 358-363. doi: 10.1016/j.neuroimage.2010.04.001
Wechsler, D. (1997). Wechsler Adult Intelligence Scale. San Antonio, TX: The Psychological Corporation.

Wu, T., Zang, Y., Wang, L., Long, X., Hallett, M., Chen, Y., et al. (2007). Aging influence on functional connectivity of the motor network in the resting state. Neurosci. Lett. 422, 164-168. doi: 10.1016/j.neulet.2007.06.011

Yang, A. C., Huang, C. C., Liu, M. E., Liou, Y. J., Hong, C. J., Lo, M. T., et al. (2014). The APOE epsilon4 allele affects complexity and functional connectivity of resting brain activity in healthy adults. Hum. Brain Mapp. 35, 3238-3248. doi: 10.1002/hbm. 22398

Yang, A. C., Huang, C. C., Yeh, H. L., Liu, M. E., Hong, C. J., Tu, P. C., et al. (2013). Complexity of spontaneous BOLD activity in default mode network is correlated with cognitive function in normal male elderly: a multiscale entropy analysis. Neurobiol. Aging 34, 428-438. doi: 10.1016/j.neurobiolaging.2012.05.004

Zhang, Y., Schuff, N., Jahng, G. H., Bayne, W., Mori, S., Schad, L. et al. (2007). Diffusion tensor imaging of cingulum fibers in mild cognitive impairment and Alzheimer disease. Neurology 68, 13-19. doi: 10.1212/01.wnl.0000250326.77323.01

Conflict of Interest Statement: The authors declare that the research was conducted in the absence of any commercial or financial relationships that could be construed as a potential conflict of interest.

Copyright (c) 2016 Yang, Tsai, Liu, Huang and Lin. This is an open-access article distributed under the terms of the Creative Commons Attribution License (CC BY). The use, distribution or reproduction in other forums is permitted, provided the original author(s) or licensor are credited and that the original publication in this journal is cited, in accordance with accepted academic practice. No use, distribution or reproduction is permitted which does not comply with these terms. 